\title{
COVID-19 vaccine willingness among patients with mental illness compared with the general population - CORRIGENDUM
}

\section{Corrigendum}

Cite this article: Jefsen $\mathrm{OH}$, Kølbæk $\mathrm{P}$, Gil Y, Speed M, Dinesen PT, Sønderskov KM, and Østergaard SD. (2022) COVID-19 vaccine willingness among patients with mental illness compared with the general population CORRIGENDUM. Acta Neuropsychiatrica 34:107. doi: 10.1017/neu.2021.43

First published online: 10 January 2022

(c) The Author(s), 2022. Published by Cambridge University Press on behalf of Scandinavian College of Neuropsychopharmacology.

\section{Oskar Hougaard Jefsen MD${ }^{1}$, Pernille Kølbæk MD, PhD ${ }^{1}$, Yael Gil Bsc.med ${ }^{1}$, Maria Speed $\mathrm{PhD}^{1}$, Peter Thisted Dinesen $\mathrm{PhD}^{2}$, Kim Mannemar Sønderskov $\mathrm{PhD}^{3}$ and Søren Dinesen Østergaard MD PhD ${ }^{1}$}

${ }^{1}$ Department of Affective Disorders, Aarhus University Hospital - Psychiatry, Aarhus, Denmark.; ${ }^{2}$ Department of Political Science, University of Copenhagen, Copenhagen, Denmark. and ${ }^{3}$ Department of Political Science, Aarhus University, Aarhus, Denmark

doi: 10.1017/neu.2021.15. Published online by Cambridge University Press 17 May 2021

The number of outpatients contacts used for weighting of vaccine willingness among patients with mental illness was incorrect. The corrected weighted estimates for vaccine willingness are listed below. The primary outcome, the proportion of patients with mental illness who were willing to receive COVID-19 vaccination, was unchanged (84.8\%) by this correction.

On page 274, the odds ratio (OR) from the logistic regression on age and vaccine willingness in the patient sample

'(OR for $\leq$ first age quartile compared to $>$ third quartile $=5.76,95 \% \mathrm{CI}=1.62 ; 22.16, \mathrm{p}=0.008$ )' should be

'(OR for $\leq$ first age quartile compared to $>$ third quartile $=5.80,95 \% \mathrm{CI}=1.63 ; 22.39, \mathrm{p}=0.008)$ '

On page 274, the OR from the logistic regression on civil status and vaccine willingness in the patient sample

' $(\mathrm{OR}=4.18,95 \% \mathrm{CI}=1.52 ; 12.02, \mathrm{p}=0.006)$ ' should be

'(OR $=4.14,95 \% \mathrm{CI}=1.50 ; 11.94, \mathrm{p}=0.007)$ '

On page 274, the OR from the logistic regression on birthplace and vaccine willingness in the patient sample ' $(\mathrm{OR}=4.50,95 \% \mathrm{CI}=1.84 ; 11.01, \mathrm{p}=0.001)$ ' should be

'(OR $=4.51,95 \% \mathrm{CI}=1.84 ; 11.04, \mathrm{p}=0.001)$ '

Additionally, the weighted estimated in Supplementary material Table 1, 4, and 5 have been corrected.

The authors regret the error.

\section{Reference}

Jefsen, O., Kølbæk, P., Gil, Y., Speed, M., Dinesen, P., Sønderskov, K., \& Østergaard, S. (2021). COVID-19 vaccine willingness amongst patients with mental illness compared with the general population. Acta Neuropsychiatrica, 33(5), 273-276. doi: 10.1017/neu.2021.15 